\title{
ANATOMIA E MICROANATOMIA DO SISTEMA REPRODUTOR DE RECTARTEMON (RECTARTEMON) DEPRESSUS (HEYNEMANN) (MOLLUSCA, GASTROPODA, STREPTAXIDAE): COMPLEXO FÁLICO
}

\author{
Mônica Picoral ${ }^{1}$ \\ José Willibaldo Thomé ${ }^{2}$
}

\begin{abstract}
ANATOMy and MiCROANATOMy OF THE REPRODUCTIVE SYSTEM OF RECTARTEMON (RECTARTEMON) DEPRESSUS (HEYNEMANN) (MOLLUSCA, GASTROPODA, STREPTAXIDAE). THE. PHALLUS COMPLEX. It is described the anatomy and microanatomy of the phallus complex of Rectartemon depressus (Heynemann, 1868). The complex is composed by a phallus, a diverticle of the phallus and an epiphallus, where the deferens duct is thrown in; the retractor muscle is splitted in two parts, inserted into the region of the epiphallus through a smaller branch, and at the extremity of the diverticle, through a longer and thiner branch. The lumen of the phallus, epiphallus and diverticle have corrugations and thorns. On the phallus the thorns are arranged in a protuberance and disposed alternately in rows, all with an aculeus form and with a chestnut collor point and a yellow basis.

KEY WORDS. Streptaxidae, Rectartemon depressus, phallus complex, anatomy, reproductive system
\end{abstract}

As primeiras referências ao sistema reprodutor de espécies de Streptaxidae são dadas por STOLICZKA (1871), quando comenta que a espécie nova Streptaxis obtusus tem muitas similaridades com as espécies dos Helicidae, e registra em breve citação um "pênis" curto.

BRUGGEN (1967) comenta que os Streptaxidae formam uma família bem definida, entre outros característicos, com um "pênis" muscular dentro de uma bainha e a existência de um apêndice "peniano" em algumas espécies.

$\mathrm{O}$ arranjo e o local de inserção do ducto deferente no complexo fálico, que pode estar completamente ou parcialmente coberto por uma bainha muscular, ou não possuí-la, bem como a ligação do músculo retrator no falo ou epifalo, apresentam variações nas espécies de Streptaxidae descritos na literatura.

O trajeto do ducto deferente nos Streptaxidae pode percorrer aproximadamente $1 / 3,2 / 3$ ou a totalidade do comprimento do falo envolto pela bainha, variando conforme a espécie. $O$ ducto deferente pode, também, em algumas espécies que não possuem o falo numa bainha, percorre-lo lateralmente desde o oviducto pela vagina

1) Museu de Ciências Naturais, Fundação Zoobotânica do Rio Grande do Sul. Caixa Postal 1188, 90001-970 Porto Alegre, Rio Grande do Sul, Brasil. Bolsista CNPq/RHAE.

2) Laboratório de Malacologia, Instituto de Biociências, Pontifícia Universidade Católica do Rio Grande do Sul. Avenida Ipiranga 6681, prédio 12-D, 90619-900 Porto Alegre, Rio Grande do Sul, Brasil. Com bolsas e auxilios do CNPq, CAPES e FAPERGS.

E-mail: thomejw@music.pucrs.br 
e o átrio genital (PFEFFer 1878; WIEGMANN 1898; BAKER 1925; DEGNER 1934; LÓPEZ \& ROCANDIO 1955; REZENDE et al. 1962; BAKER 1926 apud VENMANS 1963; BERRY 1963, 1965; ADAM 1965; VISSER 1973).

Propõe-se, neste trabalho, fornecer uma descrição detalhada da anatomia, complementada com dados microanatômicos, do complexo fálico de Rectartemon depressus (Heynemann, 1868).

\section{MATERIAL E MÉTODOS}

O material e os métodos utilizados para o estudo da anatomia e da microanatomia foram descritos em PICORAL \& THOMÉ (1993, 1996).

\section{RESULTADOS}

O complexo fálico (Figs 1-3) consiste do falo que está circundado pela bainha muscular, pelo epifalo, que recebe o ducto deferente e do divertículo do falo, que se ligam cada um na extremidade bipartida do músculo retrator.

Músculo retrator do falo (Fig. 1, mrf)

Apresenta-se como uma lâmina esbranquiçada, mais longa e mais larga que o epifalo, e com sua extremidade posterior fixada ao assoalho da câmara palial como nos demais Pulmonata, com raras exceções.

A extremidade oposta, divide-se em dois feixes: um mais curto que logo após a bifurcação, insere-se na região posterior do epifalo, próximo à penetração do ducto deferente no epifalo; o outro, um pouco mais longo, insere-se no divertículo do falo.

\section{Epifalo (Figs 1, 2, ep)}

Comprimento igual ou menor que o falo, considera-se o epifalo desde a penetração do ducto deferente até uma das extremidades posteriores bifurcadas do falo.

Apresenta-se em forma de "S" e de coloração branca, translúcida, com lume amplo, provido de algumas pregas baixas, arredondadas e dispostas alternadamente. As extremidades livres das pregas, portam minúsculos espinhos, porém em menor quantidade e mais dispersos do que no falo.

\section{Ducto deferente (Figs 1, 3, def)}

O ducto deferente origina-se da diferenciação do sulco espermático, no ovispermioducto [detalhes figuras 5, 6, 20 e 21 em PicORAL \& THOMÉ (1996)], seguindo para a frente, aderido à parede interna da bainha do falo que envolve este órgão em todo o comprimento; o ducto deferente se continua para trás, levemente aderido à parede externa do falo; depois de sair da bainha, corre ventrolateralmente na parte livre do falo e epifalo, ligando-se à extremidade posterior deste último, próximo ao músculo retrator. O lume do ducto deferente, na região onde o tecido conjuntivo é comum com o oviducto, apresenta pregas irregulares, de extremidades arredondadas. 


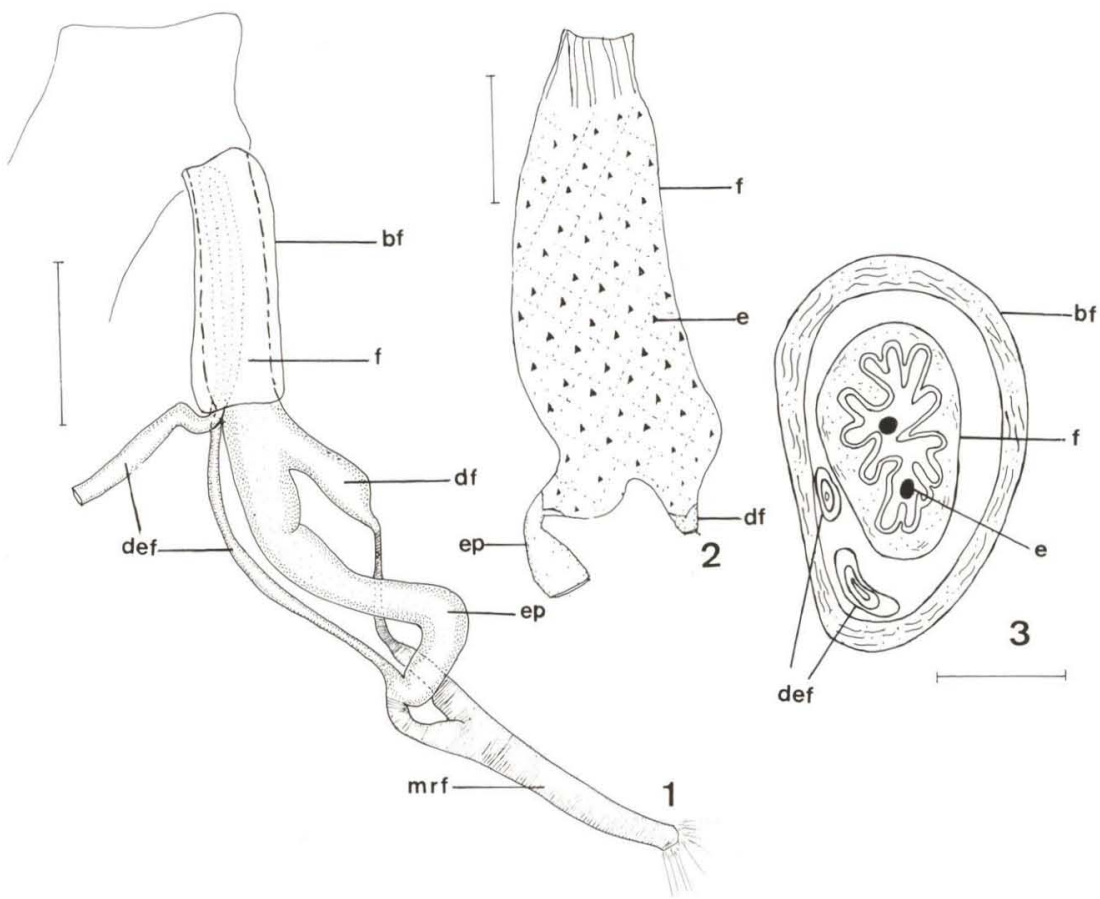

Figs 1-3. (1) Complexo fálico de Rectartemon depressus' (2) falo aberto longitudinalmente, mostrando a face interna (semi-esquemático); (3) corte transversal do falo. (bf) Bainha muscular do falo, (def) ducto deferente, (df) divertículo do falo, (e) espinho, (ep) epifalo, (f) falo, (mrf) músculo retrator do falo. Escalas: $1 \mathrm{~mm}$, Figs 1 e $2 ; 25 \mu \mathrm{m}$, Fig. 3.

A medida que o ducto deferente segue para frente, vai diminuindo de diâmetro, até chegar à metade de sua espessura na parte que volta para trás, aderido ao falo. Essa diminuição de diâmetro é acompanhada por uma diminuição do lume e o desaparecimento das pregas, apesar de um gradual aumento da túnica de fibras musculares circulares e longitudinais.

\section{Divertículo do falo (Figs 1, 2, df)}

Estrutura curta, com menos de $1 / 3$ do comprimento do falo. Está ligado ao feixe mais curto do músculo retrator e para frente continua-se pelo falo. Apresenta características similares ao falo, porém com reduzidas pregas, portando eventuais espinhos.

\section{Falo (Figs 1-3, f)}

É uma estrutura tubular, envolta parcialmente por uma bainha muscular, que se estende desde a porção posterior bifurcada do divertículo e do epifalo, finalizando no átrio genital. 
De coloração branca, levemente transparente, permitindo perceber sua estruturação interna, como um tecido rendado e lume altamente pregueado, portando espinhos.

Desde a região anterior epifálica, as pregas vão gradativamente concrescendo e apresentando o formato de um "V", obstruindo quase todo o lume do falo, na região anterior. O lume é revestido com epitélio cúbico, sustentado por tecido conjuntivo frouxo e fibras musculares.

Os espinhos fálicos (e) são córneos, cujo formato assemelha-se ao de um acúleo de roseira, tendo a base redonda e a ponta voltada para frente. Possuem coloração amarelo na base e castanho na ponta, sendo mais conspícuos e numerosos do que os da região epifálica, principalmente na porção envolta pela bainha muscular. Na porção fálica, os espinhos estão dispostos, cada um, em uma protuberância e estão arranjados em fileiras, de forma alternada (Fig. 2).

Bainha muscular do falo (Figs 1, 3, bf)

É uma grande túnica de fibras musculares circulares, revestida interna e externamente por tecido conjuntivo. Envolve mais de $2 / 3$ do falo, abrigando também parte do ducto deferente.

\section{DISCUSSÃO}

O ducto deferente que percorre totalmente a parte do falo envolto pela bainha em Rectartemon depressus, assemelha-o a Ennea insignis Pfeiffer, 1857 (PFEFFER 1878), Ennea dussumieri Férussac, 1840, Streptostele nevilli dubia Wiegmann, 1898, Streptostele nevilli H.Adams, 1868, Ennea erinaceus Martens \& Wiegmann, 1898 (WIEGMANN 1898), Gonaxis monrovia Rang, 1831, Gonaxis blacklocki Connolly, 1928, Gonaxis camerunensis Ailly, 1896 (DEGNER 1934), Streptaxis glaber Pfeiffer, 1849 (Baker 1926 apud VENMANS 1963).

O local de inserção do ducto deferente no falo, que aqui denominou-se de epifalo concorda com o descrito na literatura para as demais espécies estudadas (STOLICZKA 1871; PFEFFER 1878; WIEGMANN 1898; BAKER 1925; DEGNER 1934; STEENBERG 1936; LÓPEZ \& ROCANDIO 1955; REZENDE et al. 1962; BERRY 1963, 1965; ADAM 1965; VISSER 1973).

A divisão do músculo retrator do falo em dois feixes ocorre em Gonaxis maugerae (Gray, 1837), Edentulina liberiana (Lea, 1840), Edentulina martensi (E.A. Smith, 1882); Edentulina insignis (Pfeiffer, 1857) (DEGNER 1934) e em Streptostele buchhlotzi Martens, 1876 (LópEz \& RocANDIO 1955). No entanto, em nenhuma destas espécies o músculo retrator do falo dispõe-se dividido, com a extremidade menor ligada ao epifalo e a maior e mais delgada, ao divertículo do falo, como em Rectartemon depressus.

As espécies Ennea insignis e Streptaxis apertus Martens, 1868 (PFEFFER 1878), Ennea dussumieri, Streptostele nevilli, S.nevilli dubia (WIEGMANN 1898), Rectartemon jessei sp.n. (BAKER 1925), Gonaxis monrovia, Gonaxis blackclocki, Gonaxis camerunensis Ailly, 1897 (DEGNER 1934), Gonidomus pagoda (Férussac, 1821) (STEEnberg 1936), Edentulina oleacea (Fulton, 1903) (VERdCOURT 1961), 
Oophana diaphanopepla (Jutting, 1955)(BERRY 1963) e Streptaxis glaber Pfeiffer, 1849 (VENMANS 1963) têm semelhanças com Rectartemon depressus por apresentarem a bainha do falo cobrindo aproximadamente a metade do falo, considerando neste caso, o epifalo incluído no comprimento do falo.

Quanto ao complexo fálico, a descrição apresentada por REZENDE et al. (1962) para Streptaxis contusus (Férussac, 1821), não possibilita comparações com $R$. depressus, pois na descrição do falo não ficou claro se este está coberto total ou parcialmente pela bainha e como se constituem as porções inferior e superior do falo que recebem o músculo retrator.

Nas espécies Stenomarconia jeanneli (Germain, 1934), Gonaxis usambarensis Bequaert \& Clench, 1936, Gonaxis denticulatus (Dohrn, 1878), Gulella grossa (Martens, 1892), Gulella usagarica (Crosse, 1886) (VERDCOURT 1961), Streptostele buchhlotzi, Gulella conospira (Martens, 1892) (LóPEZ \& ROCANDIO 1955), Varicostela bequaertiana Pilsbry, 1919 (PILSBRY 1919), parece claro ocorrer um apêndice ou divertículo fálico, não havendo contudo ligação deste apêndice com o músculo retrator, como acontece com o divertículo do falo descrito em Rectartemon depressus.

Os espinhos encontrados no lume do falo de $R$. depressus também são descritos em outros gêneros de Streptaxidae. Das espécies dos gêneros Streptostele (Dohrn, 1866), Ptychotrema (Mörch, 1852), Gulella Pfeiffer, 1856, Lamelliger Ancey, 1884, Edentulina Pfeiffer, 1856, Streptaxis Gray, 1837, Gonidomus, Swainson, 1840, Sinoennea Kobelt, 1904, Discartemon Pfeiffer, 1856, Oophana Ancey, 1884, Huttonella Suter, 1890, Ennea H. \& A.Adams, 1855 (WIEGMANN 1898; DEGNER 1934; STEENBERG 1936; LóPEZ \& ROCANDIO 1955; VERDCOURT 1961, 1979, 1982; BERRY 1963, 1965; VENMANS 1963), somente as espécies de Lamelliger apresentam similaridades com Rectartemon depressus por apresentarem os espinhos com aspecto de acúleo de roseira e a ponta um pouco curvada; porém $R$. depressus diferencia-se desta e das demais espécies por apresentarem coloração amarelo na base e castanho na ponta e disposição em fileiras alternadas.

Em Rectartemon jessei, espécie tipo do gênero, confirmada por WENZ (1959) e descrito originalmente por BAKER (1925), somente a parte anterior do sistema reprodutor é mencionada, concordando com $R$. depressus quanto a descrição de um falo longo, fino, fusiforme e revestido por uma bainha. Naquela espécie não há citação de espinhos no falo, não sendo possível haver comparações, nem mesmo uma caracterização à nível genérico.

\section{CONCLUSÕES}

À nível da espécie Rectartemon depressus:

A) Propõe-se como característicos descritivos:

1) complexo fálico composto por bainha muscular, falo, epifalo, divertículo e músculo retrator;

2) $2 / 3$ do falo envolto pela bainha muscular;

3) ducto deferente penetrando sob a bainha, ascendendo a totalidade do 
comprimento do falo sob a bainha, voltando-se e descendendo sob a mesma e aderido ao falo, saindo da bainha e percorrendo o falo e epifalo até ligar-se na extremidade posterior deste, onde o músculo retrator está inserido;

4) falo com espinhos córneos no lume;

5) falo bifurcado, prolongando-se num epifalo e divertículo cujas extremidades posteriores estão ligadas ao músculo retrator.

B) Propõe-se como característicos diagnósticos:

1) músculo retrator dividido, sendo que uma das extremidades, a menor, está ligada ao epifalo e a maior e mais delgada, ao divertículo do falo;

2) falo, epifalo e divertículo apresentando espinhos córneos, sendo estes mais evidentes na região do falo. Os espinhos estão dispostos, cada um, em uma protuberância e arranjados em fileiras, de forma alternada;

3) os espinhos apresentam coloração amarelo na base e castanho na ponta.

AGRADECIMENTOS. À equipe do Instituto de Pesquisa de Planárias, UNISINOS/São Leopoldo, Rio Grande do Sul, pelo apoio técnico e uso das instalações e material histológico.

\section{REFERÊNCIAS BIBLIOGRÁFICAS}

Adam, W. 1965. LXXXV. Mollusca Streptaxidae. Annls Mus. r. Afr. cent., Tervuren, (8) 138: 1-62.

BAKER, H.B. 1925. The mollusca collected by the University of Michigan-Williamson Expedition in Venezuela. Part III. Pupilidae to Oleacinidae. Occ. Pap. Mus. Zool. Univ. Mich., Ann Arbor, 156: 1-56.

BERRY, A.J. 1963. The anatomy of two malayan limestone hill Streptaxidae, Sinoennea kanchingensis Tomlin and Oophana diaphanopepla van Benthem Jutting with special reference to the genital system. Proc. malac. Soc. Lond. 35: $139-150$.

. 1965. The genital systems of Discartemon stenostomus van Benthem

Jutting and Huttonela bicolor (Hutton) (Pulmonata; Streptaxidae) from Malayan. Proc. malac. Soc. Lond. 36: 221-228.

BRugGen, A.C. 1967. An Introduction to the Pulmonate family Streptaxidae. Jour. Conch. Lond. 26 (3): 181-188.

DEGNER, E. 1934. Westafrikanische Landschnecken. I. Streptaxiden, Helicarioniden, Vaginuliden. Zool. Jb., Abteilungen Systematik, Ökologie und Geographie der Tiere, Jena, 65: 209-308.

LÓPEZ, A.O.Z. \& A.O.Z. RocANDiO. 1955. Contribuciones al conocimiento de la fauna malacológica terrestre de la Isla de Fernando Poo. II-Família Streptaxidae.

Boln. R. Soc. esp. Hist. nat., Madrid, 53: 75-140.

Pfeffer, G. 1878. Beiträge zur Naturgeschichte der Lungenschnecken. 4. Die Agnathen. Jb. dt. Malak. Ges., Frankfurt, 5: 62-85.

Picoral, M. \& J.W. ThOMÉ. 1993. Anatomia do sistema reprodutor de Rectartemon (Rectartemon) depressus (Heynemann, 1868) (Mollusca; Gastropoda; Strepta- 
xidae). Biociências, Porto Alegre, 1 (1): 5-24.

1996. Anatomia e microanatomia do sistema reprodutor de Rectartemon

(Rectartemon) depressus (Heynemann, 1868) (Mollusca; Gastropoda; Streptaxidae): Ovispermioducto ao Poro genital. Biociências, Porto Alegre, 4 (1): 103-119.

PILSBRy, H.A. 1919. A Review of the land mollusks of the Belgian Congo Chiefly based on the collections of the American Museum Congo. Expedition 19091915. Bull. Amer. Mus. nat. Hist., New York, 40: 1-370.

Rezende, H.E.B.; P.A.F. Rodrigues \& J.L. BARros-AraúJo. 1962. Sobre o Streptaxis contusus (Férussac, 1821) (Gastropoda, Pulmonata, Streptaxidae).

Mem. Inst. Oswaldo Cruz, Rio de Janeiro, 60 (3): 337-345.

STEENBERG, C.M. 1936. Recherches anatomiques et systématiques sur le Gasteropode Pulmoné, Gonidomus pagoda (Férussac), de L'lle Maurice. Mém. Mus. r. Hist. nat. Belg., Bruxelles, 3 (2): 115-148.

StoliczKa, F. 1871. Notes on terrestrial Mollusca from the neighbourhood of Moulmein (Tenasserim Provinces), with descriptions of new species. Jour. Asiat. Soc. Beng., Calcutta, 40 (2): 143-171.

Venmans, L.A.W.C. 1963. Caribbean land Mollusca: Streptaxidae. Studies on the

Fauna of Curaçao and other Caribbean Islands, The Hague, 14: 41-76.

Verdcourt, B. 1961. Notes on the snails of North-East Tanganyika. Occ. Pap. Coryndon meml. Mus., Nairobi, 8: 1-23.

- 1979. The identity of the european greenhouse Gulella (Pulmonata: Streptaxidae). Jour. Conch. Lond. 30 (2): 101-104.

1982. Notes on east African land and freshwater snails, 12-15. Zoöl. Meded. Leiden 56 (18): 217-236.

VISSER, M.H.C. 1973. The ontogeny of the reproductive system of Gonaxis gwandaensis (Preston) (Pulmonata, Streptaxidae) with special reference to the phylogeny of the spermatic conduits of the pulmonata. Annale Univ. Stellenbosch, sér.A, Kaapstad, 48 (4): 1-79.

WENZ, W. 1959-1960. Gastropoda. Euthyneura. Berlim, Gebrüder Borntraeger, 834 p.

Wiegmann, F. 1898. II Zootomischer Theil. In: E. Martens, E. \& F. Wiegmann. Land-und Süsswasser-Mollusken der Seychellen. Mitt. Zool. Samml. Mus. Naturk. Berl., Berlin, 1 (1): 1-94.

Recebido em 16.IX.1996; aceito em 27.XI.1997. 